\section{Evaluación clínica y urodinámica de la disfunción vesical diabética}

\author{
Viveros-Elías JM, ${ }^{1}$ García-González A, ${ }^{2}$ Gaxiola-Robles ${ }^{3},{ }^{3}$ Gutiérrez-González A
}

\section{Resumen}

ANTECEDENTES: la disfunción vesical es una de las complicaciones tardías más comunes de la diabetes, con prevalencia de 20-80\%. Esta alteración afecta la calidad de vida, provoca retención, infecciones e incontinencia urinarias, incluso insuficiencia renal. Se caracteriza por disminución de la sensibilidad vesical, hiperactividad e hipocontractilidad del detrusor, y vaciamiento vesical incompleto.

OBJETIVO: evaluar si el AUA Score y la uroflujometría son efectivos para establecer el diagnóstico de disfunción vesical diabética como métodos simples, no invasivos y de bajo costo.

MATERIALES Y MÉTODOS: estudio descriptivo y trasversal, al que se incluyeron pacientes con diagnóstico de diabetes mellitus de 5 años de evolución, a quienes se les aplicó el cuestionario AUA Score, se efectuó uroflujometría (medición de orina residual) y estudio urodinámico. Se utilizó estadística descriptiva y análisis bivariado ( $U$ de Mann-Whitney, t de Student y $\chi^{2}$ ), con un nivel alfa $\mathrm{p}<0.05$.

RESULTADOS: se registraron 41 pacientes, de los que $63 \%$ tuvieron hallazgos urodinámicos de disfunción vesical diabética como: sensibilidad reducida (27\%), hiperactividad del detrusor (42\%), hipocontractilidad del detrusor (38\%) y vaciamiento incompleto (73\%). El AUA Score, Qmax en la uroflujometría libre e índice de eficiencia vesical no demostraron correlación con los hallazgos urodinámicos específicos.

CONCLUSIONES: la disfunción vesical diabética es más frecuente de lo que se piensa. Los estudios urodinámicos invasivos siguen siendo los métodos de elección para establecer el diagnóstico de disfunción vesical, como complicación de la diabetes mellitus.

PALABRAS CLAVE: disfunción vesical diabética, urodinamia, diabetes mellitus, uroflujometría, AUA Score.

Rev Mex Urol. 2017 Sep-Oct;77(5):339-345.

\section{Clinical and urodynamic evaluation of diabetic bladder dysfunction}

Viveros-Elías JM, ${ }^{1}$ García-González A, ${ }^{2}$ Gaxiola-Robles $\mathrm{R},{ }^{3}$ Gutiérrez-González A

\section{Abstract}

BACKGROUND: Diabetic bladder dysfunction is one of the most common late complications of diabetes, with a prevalence of 20$80 \%$. It affects quality of life, causing retention, infections, urinary

\footnotetext{
${ }^{1}$ Servicio de Urología.

${ }^{2}$ Servicio de Reumatología.

Central de Especialidades Médicas, La Paz, Baja California Sur, México.

${ }^{3}$ Servicio de Epidemilogía, Central de Especialidades Médicas, La Paz, Baja California Sur, México. Centro de Investigaciones Biológicas del Noroeste, Instituto Politécnico Nacional, La Paz, Baja California Sur, México.

${ }^{4}$ Departamento de Urología. Hospital Universitario, Universidad Autónoma de Nuevo León, Monterrey, México.
}

Recibido: diciembre 2016

Aceptado: julio 2017

Correspondencia

Dr. José Manuel Viveros Elías

drmanuelviveros@gmail.com

Este artículo debe citarse como

Viveros-Elías JM, García-González A, Gaxiola-Robles R, Gutiérrez-González A. Evaluación clínica y urodinámica de la disfunción vesical diabética. Rev Mex Urol. 2017 sep-oct;77(5):339-345.

DOI: https://doi.org/10.24245/revmexurol.v77i5.1116 
incontinence, and even kidney failure. Diabetic bladder dysfunction is characterized by reduced bladder sensitivity, detrusor overactivity and hypocontractility, and incomplete bladder voiding.

OBJECTIVE: The aim of our study was to evaluate whether the AUA Score and uroflowmetry were effective as simple, noninvasive, lowcost methods for diagnosing diabetic bladder dysfunction.

MATERIALS AND METHODS: A descriptive, cross-sectional study was conducted on patients diagnosed with diabetes mellitus of 5-year progression that were analyzed through the AUA Score questionnaire, uroflowmetry (residual urine measurement), and urodynamic study. Descriptive statistics and bivariate analysis (Mann-Whitney $U$ test, Student's t test, and $\chi^{2}$ test) were employed, with a $p<0.05$.

RESULTS: Forty-one patients were registered, $63 \%$ of whom had the following urodynamic findings of diabetic bladder dysfunction: reduced sensitivity (27\%), detrusor overactivity (42\%), detrusor hypocontractility (38\%), and incomplete voiding (73\%). The AUA Score, Qmax in free uroflowmetry, and Bladder Voiding Efficiency showed no correlation with the specific urodynamic findings.

CONCLUSIONS: Diabetic bladder dysfunction is more frequent than is commonly thought. Invasive urodynamic studies continue to be the basis of diagnosing bladder dysfunction as a complication of diabetes mellitus.

KEYWORDS: Diabetic bladder dysfunction; Urodynamics; Diabetes mellitus; Uroflowmetry; AUA Score

\begin{abstract}
${ }^{1}$ Servicio de Urología.
²ervicio de Reumatología.

Central de Especialidades Médicas, La Paz, Baja California Sur, México.

${ }^{3}$ Servicio de Epidemilogía, Central de Especialidades Médicas, La Paz, Baja California Sur, México. Centro de Investigaciones Biológicas del Noroeste, Instituto Politécnico Nacional, La Paz, Baja California Sur, México.

${ }^{4}$ Departamento de Urología. Hospital Universitario, Universidad Autónoma de Nuevo León, Monterrey, México.
\end{abstract}

\section{Correspondence}

Dr. José Manuel Viveros Elías drmanuelviveros@gmail.com

\section{ANTECEDENTES}

En la actualidad la diabetes mellitus ha alcanzado proporciones epidémicas importantes. En el año 2000 la prevalencia global se estimó en $2.8 \%$ para todos los grupos de edad y para el 2030 se pronostica que alcance $4.4 \%$ del total de la población mundial. ${ }^{1}$ En 2012, la Federación Internacional de Diabetes ubicó a México como uno de los países con mayor número de personas de entre 20 y 79 años de edad con diabetes mellitus, lo que se traduce en 9 millones de pacientes afectados y una prevalencia nacional de $11.9 \% .^{2}$ En América Latina este problema se magnifica al constatar que al menos un tercio de los pacientes con diabetes desconoce su enfermedad, lo que representa un desafío para el programa de detección y complica las estrategias de atención, control y prevención de la enfermedad. ${ }^{3}$

La mayoría de los pacientes con diabetes mellitus experimenta diversos cambios patológicos a intervalos variables durante la evolución de la enfermedad. Entre las complicaciones provocadas por el daño microvascular se encuentran: nefropatía, retinopatía y neuropatía diabética. ${ }^{4}$

El riesgo de neuropatía diabética es proporcional a la magnitud y duración de la hiperglucemia.., 6 Esta alteración aparece en 10-15\% de los casos al momento del diagnóstico de diabetes mellitus y en aproximadamente $50 \%$ de quienes han padecido la enfermedad por más de 25 años., De acuerdo con la Diabetes Control and Com- 
plications Trial, la neuropatía diabética puede prevenirse mediante el control óptimo de las concentraciones de glucosa sérica. ${ }^{5,7}$ La neuropatía autonómica provocada por la diabetes mellitus origina complicaciones cardiovasculares, gastrointestinales y genitourinarias; entre estas últimas están la disfunción eréctil y la disfunción vesical.

La disfunción vesical es una de las complicaciones más comunes de la diabetes mellitus, incluso supera a la nefropatía o neuropatía periférica. Hasta el momento no existen datos precisos de la incidencia y factores de riesgo provocados por la disfunción vesical diabética (cistopatía diabética); sin embargo, se ha reportado una prevalencia de 20 a $80 \%$ en pacientes con diabetes mellitus, incluso algunos autores señalan límites de 2 a $83 \%$, comparada con $60 \%$ para neuropatía periférica y $50 \%$ para nefropatía. ${ }^{8}$ La disfunción vesical diabética afecta a pacientes de uno y otro sexo, y su prevalencia aumenta con el tiempo de manifestación de la diabetes mellitus, incluso de $50 \%$ para los pacientes con 25 o más años de la enfermedad versus $10 \%$ al momento del diagnóstico. ${ }^{6} \mathrm{La}$ mayoría de los pacientes con disfunción vesical diabética suele tener complicaciones a largo plazo por la diabetes mellitus.

Los síntomas de disfunción vesical diabética son insidiosos y se caracterizan por insuficiencia progresiva de la capacidad de vaciado de la vejiga. La sensibilidad propioceptiva del volumen intravesical suele alterarse en etapas tempranas de la enfermedad. Los pacientes inician con retraso de la micción, concomitante con menor sensibilidad, prolongan los intervalos intermiccionales y tienen dificultad para el vaciamiento vesical, además de aumentar la cantidad de orina residual. ${ }^{9}$ Esto puede provocar retención urinaria completa con incontinencia por rebosamiento, incluso insuficiencia renal. La hiperactividad del detrusor (urodinámicamente determinada por contracciones no inhibidas durante el llenado) y la hiperactividad vesical (clínicamente determinada por urgencia con o sin incontinencia, polaquiuria y nicturia $)^{10}$ suelen aparecer en los pacientes con disfunción vesical diabética antes que la hipocontractilidad del detrusor, incluso se ha descrito que pueden ser más frecuentes. ${ }^{11}$

El diagnóstico de disfunción vesical diabética depende, hasta el día de hoy, de los estudios urodinámicos invasivos, pues algunas instituciones médicas no cuentan con el equipo necesario, además de ser costosos. La uroflujometría es un estudio no invasivo, relativamente económico y de mayor accesibilidad.

El objetivo de este estudio fue: evaluar si el cuestionario AUA Score y la uroflujometría con medición de orina residual, para determinar el índice de eficiencia vesical (IEV), son capaces de establecer el diagnóstico de disfunción vesical diabética, pues representan métodos simples, no invasivos y de bajo costo, para de esta manera hacer más accesible la detección del trastorno en la práctica clínica diaria.

\section{MATERIALES Y MÉTODOS}

Estudio descriptivo y trasversal, al que se incluyeron pacientes adultos que acudieron a la consulta externa de Urología de la Central de Especialidades Médicas de La Paz, Baja California Sur, de julio de 2014 a septiembre de 2015, con diagnóstico de diabetes mellitus de 5 años de evolución, como periodo mínimo de manifestación. Criterios de inclusión: pacientes sin infección urinaria activa al momento del estudio y que hubieran aceptado firmar el consentimiento informado. Criterios de exclusión: pacientes con alteración prostática grado II o III al examen digital rectal, o mayor de 40 g por ultrasonido suprapúbico. Además, mujeres con cistocele posiblemente obstructivo. También se excluyeron los pacientes con alteraciones neu- 
rológicas diferentes a la neuropatía diabética o con trastornos no neurológicos que afectaran la función vesical (antecedente de histerectomía, cirugía pélvica o radioterapia).

A todos los pacientes se les aplicó el cuestionario AUA Score, que incluye 7 ítems de: pujo, tenesmo, chorro débil, intermitencia, intervalo miccional mayor de 2 horas, urgencia y nicturia, además de una pregunta adicional de calidad de vida. Se clasificaron como síntomas leves los resultados de las puntuaciones de 0-7, síntomas moderados de 8-19 y síntomas severos de 2035 obtenidas en el AUA Score. ${ }^{12}$ Posteriormente se realizó una uroflujometría libre y se midió la orina residual, mediante la colocación del catéter de cistometría, para determinar el índice de eficiencia vesical (IEV = Volumen vaciado / capacidad vesical total X 100). ${ }^{13}$ En seguida se efectuó el estudio urodinámico, con cistometría de llenado, prueba de presión de punto de fuga (ALPP) para los pacientes con incontinencia urinaria de esfuerzo y, al llegar a la capacidad cistométrica máxima, se realizó el estudio de presión-flujo. A todos los pacientes se les realizó electromiografía simultánea, como parte del estudio urodinámico completo. Se utilizaron las definiciones estandarizadas de la International Continence Society ${ }^{10}$ y de "Buenas Prácticas Urodinámicas" de la misma. ${ }^{14}$ En la uroflujometría se consideró flujo bajo al valor de Qmax menor de $15 \mathrm{~mL} / \mathrm{seg}$; eficiencia vesical disminuida al índice de eficiencia vesical menor de $75 \%$, vaciamiento vesical incompleto cuando la orina residual posmiccional superó $10 \%$ del volumen premiccional o cuando el volumen de orina residual superó $100 \mathrm{~mL}$. En el estudio urodinámico invasivo se consideró disfunción vesical diabética cualquiera de los siguientes hallazgos: sensibilidad vesical reducida (primer deseo miccional abolido o posterior a la infusión de 300 $\mathrm{mL}$ ), hiperactividad del detrusor (contracciones no inhibidas durante el llenado vesical mayores de $5 \mathrm{~cm} \mathrm{H}_{2}$ ) o hipocontractilidad del detrusor al encontrar un índice de contractilidad (IC =
5 x Qmax + Pdet@Qmax) menor de 100, que se traduce en flujo máximo bajo y presión del detrusor baja al flujo máximo. ${ }^{13}$

Para el análisis estadístico se utilizó el programa SPSS para Windows, versión 15.0, con estadística descriptiva y análisis bivariado $(U$ de Mann-Whitney, t de Student y $\chi^{2}$ ). Se consideró estadísticamente significativo el valor alfa $p>0.05$.

\section{RESULTADOS}

Se registraron 41 pacientes: 24 mujeres y 17 hombres, con edad promedio de 60.8 años con 14.5 años de habérseles establecido el diagnóstico de diabetes mellitus (5 a 36 años). Se encontró disfunción vesical diabética en 63\% (26/41) de los pacientes. Los hallazgos urodinámicos más frecuentes fueron: sensibilidad reducida en $27 \%$ (7/26), hiperactividad del detrusor en $42 \%$ (11/26), hipocontractilidad del detrusor en 38\% (10/26) y vaciamiento incompleto en $73 \%$ (17/26) de los casos. De los 15 pacientes sin disfunción vesical diabética, 24\% (4) reportaron obstrucción infravesical, todos de sexo masculino (Cuadro 1).

El promedio de puntuación del AUA Score de los pacientes sin disfunción vesical diabética fue de 8.67; se clasificaron con síntomas leves $60 \%$ (9), moderados $26 \%$ (4) y severos $13 \%$ (2) de los casos; el promedio de Qmax fue de $19.49 \mathrm{~mL} / \mathrm{seg}$ y $33 \%$ (5) tuvieron Qmax menor de $15 \mathrm{~mL} / \mathrm{seg}$.

EI AUA Score de los pacientes con disfunción vesical diabética fue de 10.44; el promedio de Qmax fue de $20.41 \mathrm{~mL} / \mathrm{seg}$ y $30 \%$ (8) reportaron un Qmax menor de $15 \mathrm{~mL} / \mathrm{seg}$. Se clasificaron con síntomas leves (AUA Score 0-7) 44\% (11), síntomas moderados (AUA Score 8-19) 40\% (10) y síntomas severos (AUA Score 20-35) 16\% (4) de los pacientes. Al analizar el índice de eficiencia vesical (IEV) menor de $75 \%$ se reportaron $20 \%$ (3) versus $50 \%$ (12) de casos sin y con disfunción vesical diabética, respectivamente (Cuadro 2). 


\begin{tabular}{|c|c|c|c|}
\hline & Masculino $(n=17)$ & Femenino $(n=24)$ & Total $(n=41)$ \\
\hline Con disfunción vesical diabética & $34 \%(9)$ & $65 \%(17)$ & $63 \%(26)$ \\
\hline Sensibilidad vesical reducida & $71 \%(5)$ & $28 \%(2)$ & $27 \%(7)$ \\
\hline Hiperactividad del detrusor & $27 \%(3)$ & $73 \%(8)$ & $42 \%(11)$ \\
\hline Hipocontractilidad del detrusor & $40 \%(4)$ & $60 \%(6)$ & $38 \%(10)$ \\
\hline Vaciamiento incompleto & $12 \%(2)$ & $88 \%(15)$ & $73 \%(17)$ \\
\hline Sin disfunción vesical diabética & $60 \%(9)$ & $40 \%(6)$ & $37 \%(15)$ \\
\hline Función vesical normal & $45 \%(5)$ & $54 \%(6)$ & $73 \%(11)$ \\
\hline Obstrucción infravesical & $24 \%(4)$ & - & $24 \%(4)$ \\
\hline
\end{tabular}

El análisis bivariado no reportó diferencias estadísticamente significativas inter o intragrupales. Los modelos de regresión logística (mediante "stepwise") tampoco mostraron asociación entre la disfunción vesical diabética y cualquiera de los ítems del AUA Score, con el Qmax promedio de la uroflujometría libre, el Qmax menor o mayor de $15 \mathrm{~mL} / \mathrm{seg}$ y el índice de eficiencia vesical (IEV) menor y mayor de $75 \%$.

\section{DISCUSIÓN}

La disfunción vesical diabética es más frecuente de lo que se piensa, pues se encontró en $63 \%$ de los pacientes con más de 5 años de habérseles establecido el diagnóstico de diabetes mellitus, lo que representa una prevalencia similar a la de estudios previos (20 a 80\%). ${ }^{8}$ En nuestra serie, la manifestación urodinámica más frecuente de la disfunción vesical diabética fue el vaciamiento incompleto (73\%), como resultado de la hipocontractilidad del detrusor, ya sea de fuerza o duración. Así mismo, se observó hipocontractilidad del detrusor (de fuerza) en $38 \%$ e hiperactividad del detrusor en $42 \%$ de los casos. Estos resultados coinciden con los reportados en otras series. Kaplan y su grupo ${ }^{11}$ encontraron hiperactividad del detrusor en $55 \%$ de los pacientes, seguido de hipocontractilidad del detrusor en $33 \%$, además de distensibilidad

Cuadro 2. AUA Score, Qmax, índice de eficiencia vesical y disfunción vesical diabética

\begin{tabular}{|c|c|c|c|}
\hline Puntuación & $\begin{array}{l}\text { Sin DVD } \\
(n=15) *\end{array}$ & $\begin{array}{l}\text { Con DVD } \\
(n=26)^{* *}\end{array}$ & $\mathbf{p}$ \\
\hline AUA Score 0-7 & $60 \%(9 / 15)$ & $44 \%(11 / 26)$ & \\
\hline AUA Score 8-19 & $26 \%(4 / 15)$ & $40 \%(10 / 26)$ & \\
\hline AUA Score 20-35 & $13 \%(2 / 15)$ & $16 \%(4 / 26)$ & \\
\hline Qmax promedio & $19.49 \mathrm{~mL} / \mathrm{seg}$ & $20.41 \mathrm{~mL} / \mathrm{seg}$ & $\begin{array}{c}p=0.76 \\
U=169.5\end{array}$ \\
\hline Qmax $<15 \mathrm{~mL} / \mathrm{seg}$ & $33 \%(5 / 15)$ & $30 \%(8 / 26)$ & $\begin{array}{l}p=0.41 \\
U=17.0\end{array}$ \\
\hline IEV $>75 \%$ & $80 \%(12 / 15)$ & $50 \%(13 / 26)$ & \\
\hline IEV $<75 \%$ & $20 \%(3 / 15)$ & $50 \%(13 / 26)$ & $\begin{array}{l}p=0.06 \\
\chi^{2}=3.5\end{array}$ \\
\hline
\end{tabular}

DVD: disfunción vesical diabética; Qmax: flujo máximo en uroflujometría libre; IEV: índice de eficiencia vesical. AUA Score total promedio: * $\sin$ DVD: 8.67 puntos. ${ }^{* *}$ con DVD: 10.44 puntos. 
vesical baja en $15 \%$ como hallazgo adicional. Los pacientes con diabetes manifiestan síntomas urinarios (llenado y vaciamiento vesical) que repercuten en la calidad de vida, pero no son excluyentes; por lo tanto, un paciente con vaciamiento incompleto puede tener hiperactividad vesical e incontinencia de urgencia concomitantes. ${ }^{15}$

En nuestro estudio no encontramos diferencias significativas entre el puntaje del AUA Score y el Qmax de los pacientes con y sin disfunción vesical diabética. Los síntomas iniciales de esta alteración pueden pasar inadvertidos por el paciente, pues su manifestación es insidiosa. En la consulta médica debe efectuarse el interrogatorio directo, con la finalidad de que el paciente se percate de su coexistencia. Así mismo, son inespecíficos a cierto comportamiento vésico-uretral e incapaces de diferenciarse de otras alteraciones, principalmente la obstrucción infravesical. Groutz y sus colaboradores ${ }^{16}$ evaluaron la correlación entre el AUA Score y los hallazgos urodinámicos en 587 mujeres, a quienes se les realizaron estudios urodinámicos por diferentes motivos. Encontraron un índice significativamente mayor en el grupo con obstrucción, clasificándose como síntomas severos en 34 versus $7 \%$ del grupo control. Sin embargo, no se encontró correlación entre las evaluaciones del AUA Score y los hallazgos urodinámicos asociados con el objetivo del estudio. También concluyeron que el AUA Score puede utilizarse como índice de incomodidad con los síntomas urinarios de la vía inferior en mujeres con obstrucción infravesical. No obstante, los síntomas evaluados en el AUA Score son subjetivos e inespecíficos, por lo que la evaluación urodinámica completa es esencial para establecer el diagnóstico.

En nuestra serie, el Qmax obtenido en la uroflujometría no invasiva no demostró ser efectivo para sospechar la disfunción vesical diabética, quizás porque de manera compensatoria el paciente con hipocontractilidad del detrusor utiliza la musculatura abdominal para asistir el vaciamiento vesical y la medición del Qmax no puede demostrar la contracción propia del detrusor, excepto cuando se acompaña de la medición de la presión abdominal y vesical, simultáneamente, durante la micción. De la misma manera, el índice de eficiencia vesical, por sí solo, fue incapaz de establecer el diagnóstico de disfunción vesical diabética, debido a que la presión abdominal ejercida por la musculatura abdominal puede contribuir al vaciamiento $y$, por lo tanto, sesgar los resultados. El estudio de Lee y sus coautores, ${ }^{17}$ en el que evaluaron a 182 mujeres mediante el AUA Score, uroflujometría y medición de orina residual a través de ultrasonido y cateterismo, definieron la disfunción vesical diabética como una capacidad vesical mayor de $500 \mathrm{~mL}$, orina residual mayor de $100 \mathrm{~mL}$ y en pacientes con orina residual entre 50 y $100 \mathrm{~mL}$, su punto de corte tuvo una eficiencia vesical menor a $75 \%$. Encontraron disfunción vesical diabética en $25.8 \%$ (47/182) de las pacientes y de éstas, 57\% (27/47) tuvo orina residual superior a $100 \mathrm{~mL}$ y $36 \%$ (17/47) orina residual entre 50 y $100 \mathrm{~mL}$, con eficiencia vesical menor de $75 \%$. Sin embargo, los autores no contrastaron los resultados contra el patrón de referencia, es decir, el estudio presión-flujo. Además, creemos que no es suficiente evaluar solamente el Qmax y la eficiencia vesical como parámetro de disfunción vesical diabética, ya que sólo evalúa el vaciamiento incompleto o la eficiencia vesical disminuida, pasando inadvertidos los datos de disfunción vesical durante la fase de llenado.

\section{CONCLUSIÓN}

El AUA Score, la uroflujometría no invasiva y la medición de orina residual son incapaces de establecer el diagnóstico de disfunción vesical diabética; por tanto, debe tenerse un alto índice de sospecha de su manifestación. Los estudios 
urodinámicos invasivos (cistometría de llenado y estudio de presión-flujo) siguen siendo el método de elección para diagnosticar esta complicación tardía de la diabetes mellitus.

\section{Agradecimientos}

Agradecemos la colaboración de la Dra. María de Jesús Peña Morán por su apoyo en la realización de este estudio.

\section{Financiamiento}

Los autores no recibieron ningún patrocinio para llevar a cabo este estudio.

\section{Conflicto de interés}

Los autores declaran no tener conflicto de intereses.

\section{REFERENCIAS}

1. Wild S, Roglic G, Green A, et.al. Global prevalence of diabetes. Estimates for the year 2000 and projections for 2030. Diabetes Care. 2004;27:1047-1053.

2. Instituto Nacional de Salud Pública. Encuesta Nacional de Salud y Nutrición 2012. México. 2012;108-122.

3. Rebolledo FA. Neuropatía diabética. Aspectos prácticos, diagnóstico, terpéuticos y medidas profilácticas. $2^{\underline{a}}$ ed. México: Editorial Alfil, 2008;XIII-XXVII.

4. Jung HS. Clinical implications of glucose variability: chronic complications of diabetes. Endocrinol Metab. 2015;30(2):167-74.

5. Boulton A, Vinik A, Arezzo J, et al. Diabetic neuropathies. Diabetes Care. 2005;28 (4):956-962.
6. Jensen D, Klevmark V. Systemic illnesses (diabetes mellitus, sarcoidosis, alcoholism, and porphyrias). Diabetes mellitus. Corcos J, Schick E. Textbook of Neurogenic Bladder. 2ª ed. Londres: Informa Healthcare, 2008;229-233.

7. Sheng $C$, Huai $T$, Hong Y. Are lower urinary tract symptoms in women with Type 2 Diabetes affected by glycemic control?. J Urol. 2013;189(4S):e649

8. Gomez CS, Kanagarajah P, Gousse A. Bladder dysfunction in patients with diabetes. Curr Urol Rep 2011;(12):419426.

9. Yamaguchi C, Sakakibara R, Uchiyama T, et al. Bladder sensation in peripheral nerve lesions. Neurourol Urodyn. 2006;25:763-9.

10. Abrams P, Cardozo L, Fall M, Griffiths D, et al. The standardisation of terminology of lower urinary tract function: report from the Standardisation Sub-committee of the International Continence Society. Neurourol Urodyn. 2002;21(2):167-78.

11. Kaplan S, Te A, Blaivas JG, et al. Urodynamic findings in patients with diabetic cystopathy. J Urol. 1995;153(2):342344.

12. Kaplan S. Update on the American Urological Association Guidelines for the Treatment of Benign Prostatic Hyperplasia. Rev Urol. 2006;8(suppl 4):S10-S17.

13. Abrams P. Bladder Outlet Obstruction Index, Bladder Contractility Index and Bladder Voiding Efficiency: three simple indices to define bladder voiding function. BJU Int 1999;84(1):14-15.

14. Shäfer W, Abrams P, Liao L, et al. Good urodynamic practices: uroflowmetry, filling cystometry, and pressure-flow studies. Neurourol Urodyn 2002;21(3):261-74.

15. Daneshgari F, Guiming L, Lori Birder, et al. Diabetic Bladder Dysfunction: Current Translational Knowledge. J Urol 2009;182;S18-S-26.

16. Groutz A, Blaivas J, Fait G, et al. The significance of the American Urological Association symptom index score in the evaluation of women with bladder outlet obstruction. J Urol. 2000;163(1):207-11.

17. Lee WC, Wu CC, Wu HP, Tai TY. Lower urinary tract symptoms and uroflowmetry in women with type 2 diabetes mellitus with and without bladder dysfunction. Urology. 2007;69(4):685-90. 